\title{
Diseño y desarrollo de lecciones mediante el enfoque del currículo costarricense de Matemática
}

\section{Lesson design and development using the Costa Rican Mathematics curriculum}

\author{
Luis Armando Hernández Solís \\ Académico, Escuela de Ciencias Naturales y Exactas \\ Universidad Estatal a Distancia \\ San José, Costa Rica \\ Ihernandez@uned.ac.cr
}

Recibido - Received: 11 / 01 / $2020 \quad$ Corregido - Revised: 10 / 02 / 2020 Aceptado - Accepted: 21 / 02 / 2020

DOI: https://doi.org/10.22458/ie.v22i32.2767

URL: https://revistas.uned.ac.cr/index.php/innovaciones

\begin{abstract}
Resumen: La resolución de problemas ha sido incorporada de distintas formas en los planes de estudio de varios países; en Costa Rica se coloca como enfoque principal del currículo nacional con un carácter metodológico que orienta la acción de aula y estructura la lección. El artículo describe el diseño de dos tareas matemáticas formuladas en un problema geométrico cuyo propósito educativo es la introducción del concepto de bisectriz y de una propiedad asociada a esta. Se realiza para cada tarea un análisis de activación de procesos matemáticos para luego contrarrestarlos con la evidencia recolectada en la aplicación del problema a un grupo de primer año de educación secundaria del Colegio Técnico Profesional de Pacayas, Cartago, Costa Rica. Asimismo, un balance global del desarrollo de la lección para evaluar si el problema planteado cumplió con los propósitos educativos establecidos previamente. Se concluye que el estudio ofrece a la comunidad educativa una experiencia exitosa en el diseño y ejecución de una lección enmarcada en el enfoque de resolución de problemas para el aprendizaje de conocimientos nuevos, acorde con los programas de estudio del Ministerio de Educación Pública de Costa Rica MEP (2012)
\end{abstract}

Palabras clave: Enseñanza de las matemáticas, Enseñanza secundaria (1er nivel), Geometría, Razonamiento, Reforma educativa, Resolución de problemas

Summary: Problem solving has been incorporated in different ways into the curricula of various countries. In Costa Rica, it is placed as the main focus of the national curriculum with a methodological character that guides classroom action and structures the lesson. The article describes the design of two mathematical tasks formulated in a geometric problem the educational purpose of which is the introduction of the concept of bisector and its associated property. An analysis of the activation of mathematical processes is carried out for each task to then counter them with the evidence collected when applying the problem to a group of first year of secondary education of the Professional Technical High-School of Pacayas, Cartago, Costa Rica. Also, a global balance is made of how the lesson develops to assess if the problem posed fulfilled the previously established educational purposes. The conclusion is that the study offers the educational community a successful experience in the design and execution of a lesson framed in the problem-solving approach for learning new knowledge, in accordance with the study programs of the Ministry of Public Education of Costa Rica. MEP (2012).

Key Words: Teaching Mathematics, Secondary education (1st level), Geometry, Reasoning, Educational reform, Problem solving 


\section{INTRODUCCIÓN}

A partir de Polya (1945), la resolución de problemas ha tomado gran relevancia en el mundo, siendo un referente para diversas investigaciones y parte central de currículos escolares orientales como los de Japón, Corea y Singapur. Según Isoda y Katagiri (2016): “En los años 60 fue señalado como el enfoque de enseñanza para desarrollar pensamiento matemático que se recomendaba en el desarrollo de pensamiento de alto nivel para la formación del carácter humano" (p. 25). En Costa Rica, aunque desde 1995 se propuso dar prioridad a la "resolución de problemas", no trascendió en la acción de aula. A partir del 2012, se colocó la resolución de problemas como enfoque principal del currículo nacional con un carácter metodológico que orienta la acción de aula y estructura la lección.

Sin embargo, en la educación secundaria de Costa Rica ha prevalecido una gestión de aula orientada a la "transmisión" de conocimientos, en la asignatura de Matemática la acción docente se ha centrado mayoritariamente en explicar algoritmos, establecer definiciones, teoremas y ejemplos de ejercicios a un grupo de estudiantes que se encargan de repetir mecánicamente esos procedimientos en tareas rutinarias y muy similares. Así lo indica el MEP (2012, p. 498): "Lo que ha predominado en la educación secundaria del país es un estilo de organización de la lección que subraya una secuencia de pasos o momentos: Teoría->Ejemplo->Práctica Rutinaria, y a veces la introducción como apéndice de un ejercicio contextualizado".

En el 2013, inició el proceso de implementación curricular en la asignatura de Matemática para educación primaria y secundaria, lo cual ha provocado algunos desafíos a la labor docente; entre ellos la preparación de las lecciones. El Programa Estado de la Nación (PEN), ente costarricense dedicado a la investigación participativa para la promoción del desarrollo humano sostenible, en su Quinto Informe del Estado de la Educación señala: “La preparación de la acción de aula adquiere un lugar más relevante con este currículo que con los anteriores. Demanda de los docentes una mayor preparación en los diversos aspectos pedagógicos y cognoscitivos presentes en la lección" (2015, p. 156).

Aunque el currículo plantea cuatro momentos en la organización de la lección, es importante subrayar que la primera fase Propuesta de un problema es clave para desencadenar la construcción o la movilización de los aprendizajes, MEP (2012, p.13): "Aprender a plantear y resolver problemas y especialmente usarlos en la organización de las lecciones se adopta como la estrategia central para generar esas capacidades". Del mismo modo Ruiz $(2018$, p.77) apunta "[...] el corazón de la orientación metodológica general que plantea el currículo es el diseño apropiado de problemas o tareas matemáticas por medio de los cuales en el aula se logre potenciar el desarrollo de la competencia matemática".

Por tanto, con el nuevo enfoque curricular era necesario un cambio en la preparación de la lección no solo en estructura, sino también en el énfasis y relevancia que se le debe dar a la selección y diseño de problemas. Asimismo, el nuevo paradigma exige un cambio en el rol docente, de transmisor a facilitador del conocimiento; no obstante, Meza, Agüero y Suárez (2019) apuntan que en el aula de Matemática siguen prevaleciendo lecciones expositivas de carácter tradicional.

Aunque se han publicado propuestas de modelos de lección que incorporan la organización de la lección y el enfoque de resolución de problemas propuesto por MEP (2012), tanto para educación primaria (Zumbado, Oviedo y Vargas, 2015, p. 275) como educación secundaria (Hernández y López, 2015, p. 289), se considera fundamental en este proceso de implementación que surjan nuevas iniciativas por parte de los docentes e investigadores. Blanco, Martínez y Jiménez (2018) señalan que existe una demanda explícita de investigaciones que apoyen de forma directa los procesos de enseñanza y aprendizaje, y que no solo se queden en la periferia de la práctica sin llegar al aula. 
A continuación, se ofrece a la comunidad educativa una experiencia en el diseño y puesta en práctica de un problema para el aprendizaje de conocimientos nuevos en lecciones de Matemática, enmarcadas en el enfoque metodológico de resolución de problemas acorde con los programas de estudio MEP (2012).

\section{MARCO TEÓRICO}

Existen varias formas de entender la resolución de problemas. Por ejemplo, el enfoque japonés tiene una orientación pragmática que influye directamente en la acción de aula, en otros casos la perspectiva es sobre todo el aprendizaje de estrategias resolutorias. La resolución de problemas como estrategia metodológica pretende que el estudiante se enfrente a una situación en donde se planteen interrogantes, y en el proceso de solución pueda compartir y discutir sus hallazgos y estrategias, logrando así una mayor interacción social y construcción de los significados. Desde esta perspectiva, el estudiante no debe ser concebido como un sujeto que sigue una serie de pasos para resolver problemas, sino como el sujeto que activa procesos de pensamiento en la búsqueda de estrategias de solución efectivas (Díaz y Díaz, 2018).

En la literatura se encuentran diferentes propuestas referentes a los pasos por seguir en la resolución de un problema de acuerdo con la naturaleza del problema y del contexto educativo (e. g., Polya, 1945; Mason, Burton y Stacey, 1982; Schöenfeld, 1985; Bransford y Stein, 1987; de Guzmán 1991; Yerushalmy, 2000; Villegas, Castro y Gutiérrez, 2009; Yimer y Ellerton, 2010).

Prácticamente, los modelos son muy similares y responden a las cuatro fases establecidas por Polya (1945): comprensión del problema, diseño de un plan de acción, ejecución del plan y la reconsideración o retrospección.

Con una perspectiva que se aproxima al enfoque japonés, el currículo costarricense establece un estilo de acción de aula con una estructura que contempla cuatro momentos centrales: propuesta de un problema, trabajo estudiantil independiente, discusión interactiva y comunicativa y la clausura o cierre.

Asimismo, el esquema de lección costarricense emplea dos etapas diferentes. La primera busca generar conocimientos nuevos y la segunda ocurre una vez realizada la primera, y busca reforzar y ampliar el papel de los aprendizajes desarrollados. Para ambas etapas, la escogencia del problema inicial es transcendental para el logro de los propósitos de enseñanza. Pero, ¿cuándo una tarea matemática se considera un problema?

El MEP (2012, p. 29) define un problema como “[...] un planteamiento o una tarea que busca generar la interrogación y la acción estudiantil utilizando conceptos o métodos matemáticos". Isoda y Olfos (2009) señalan:

El verdadero problema es aquel que pone al alumno en una situación nueva, ante la cual no dispone de procedimiento inmediato para su resolución. Por ende, un problema se define en cuanto a su relación con el sujeto que lo enfrenta y no en cuanto a sus propiedades intrínsecas. (p. 99)

Es decir, un problema no depende de la tarea matemática vinculada a él, sino de la relación que existe entre la persona y la tarea; es decir, si cuenta o no con los conocimientos para resolverlo de forma inmediata. Un problema puede ser un ejercicio para un individuo que haya resuelto tareas matemáticas similares; por lo tanto, un problema debe presentar un desafío, sin ser un problema inaccesible. Mazzilli, Hernández y De La Hoz (2016), luego de revisión bibliográfica sistemática extrajeron las siguientes características para un problema que se resumen en tres aspectos: 
- El desconocimiento de la vía o camino de solución.

- La no existencia de una solución inmediata.

- La existencia de un interés para transformar la situación a la cual se enfrentan.

Con el propósito de generar oportunidades para promover capacidades superiores en los estudiantes, el currículo costarricense de Matemáticas plantea tres niveles de complejidad para las tareas matemáticas: reproducción, conexión y reflexión. Estos son similares a los establecidos por la Organización para la Cooperación y el Desarrollo Económicos OCDE en el 2003, pero con una perspectiva más global que la evaluación de los aprendizajes.

Cada nivel está íntimamente ligado al grado de la activación de procesos matemáticos: "Actividades cognitivas (o tipos de actividades) que realizan las personas en las distintas áreas matemáticas y que se asocian a capacidades para la comprensión y uso de los conocimientos" (MEP, 2012, p. 475). La activación de estos procesos transversales en la acción de aula apoya el progreso de diversas dimensiones de la competencia matemática. El modelo propuesto por Ruiz (2018) plantea que estos procesos matemáticos (o capacidades que implican) determinan los niveles de complejidad; es decir, si se establece el papel preciso de intervención de esos procesos en una tarea matemática, será posible identificar el nivel de complejidad.

\section{METODOLOGÍA}

El enfoque de la investigación es interpretativo ya que se centra, principalmente, en comprender los fenómenos educativos a través del análisis de elementos cualitativos reflejados en una determinada acción educativa (Gil, León, y Morales, 2017; Cerrón, 2019). Asimismo, la investigación es de tipo exploratorio, pues la muestra es intencional y moderada en tamaño; además, es una investigación aplicada ya que su propósito es brindar pautas prácticas respecto al diseño y validación de tareas matemáticas a partir de una experiencia (Vargas, 2009).

En síntesis la investigación se desarrolló en cinco etapas que se resumen a continuación:

\section{Diseño del problema y plan de lección}

El problema se elaboró a partir de las definiciones de Isoda y Olfos (2009) y del MEP (2012), tomando en cuenta las características establecidas en Mazzilli, Hernández y De La Hoz (2016); y con el propósito de propiciar el desarrollo de la habilidad específica "Determinar medidas de ángulos sabiendo que son congruentes, complementarios o suplementarios con otros ángulos dados" (MEP, 2012, p. 303).

Para el diseño del problema se consideraron las habilidades específicas antes desarrolladas que podrían servir de "andamios" para su resolución, que de acuerdo con el MEP (2012, p. 303) serían:

- Reconocer en diferentes contextos ángulos llanos, adyacentes, los que forman par lineal y los opuestos por el vértice.

- Identificar ángulos congruentes, complementarios, suplementarios en diferentes contextos.

En síntesis, el problema posee un contexto matemático e intenta introducir el concepto de bisectriz de un ángulo a través de la exploración de una situación geométrica particular en donde se plantea intrínsecamente la propiedad: Si dos rectas se intersecan, las bisectrices de una pareja de ángulos consecutivos, forman un ángulo recto. Ver figura 1. 
Figura 1. Representación gráfica de la propiedad por explorar.

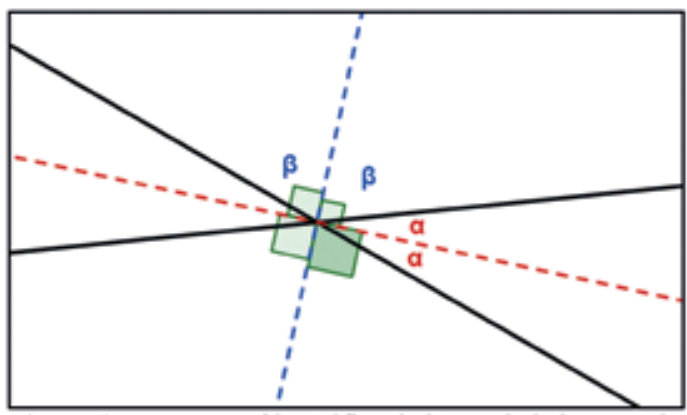

Fuente: Elaboración propia (2019).

El problema (anexo A) presenta dos tareas matemáticas, en la primera se solicita la medida del ángulo FEG de manera que se cumplan las características geométricas dadas en la representación gráfica, y en la segunda tarea se pide una generalización de la medida del ángulo FEG si las medidas de los ángulos a y $\beta$ varían.

Ambas tareas se pueden resolver empleando ya sea una estrategia deductiva o una inductiva. Isoda y Olfos (2009, p. 100) indican "[...] son buenos aquellos problemas que admiten varios enfoques para su resolución, tanto intuitivos como formales". Por ejemplo, el estudiante podría identificar que el ángulo $A E B$ es llano y está conformado por lo ángulos consecutivos $A E C$ y CEB, con lo que se tendría que $2 \alpha+2 \beta=180^{\circ}$, entonces FEG sería recto debido a que su medida es igual a $\alpha+\beta$. Pero también el estudiante podría optar por una estrategia inductiva asignando diferentes medidas a los ángulos (a y $\beta$ ) de tal forma que que cumplan con los supuestos geométricos establecidos, y paulatinamente ir descubriendo algún patrón que pudiera generalizar.

El problema está asociado a un plan de lección referente al área de Geometría, para ser empleado en la etapa 1 (aprendizaje de conocimientos nuevos), para esto se utilizó como referencia el modelo de clase basada en el método de resolución de problemas propuesto por Isoda Arcavi y Mena (2012, p. 117), ver anexo B. En la tabla 1 se realiza una síntesis de los momentos que se planificaron en la clase de dos lecciones consecutivas de 40 minutos cada una.

TABLA 1

Resumen de momentos de la clase

\section{Propuesta de un problema (15 minutos)}

En un primer momento los estudiantes leen de forma individual la hoja de trabajo entregada, luego, el docente expone la situación de aprendizaje correspondiente $y$, sin dar posibles caminos o estrategias, se asegura mediante preguntas dirigidas que se comprende correctamente el problema. Por ejemplo, si comprende qué son rectas concurrentes y la simbología que se emplea para denotar los ángulos congruentes.

\section{Trabajo estudiantil independiente (30 minutos)}

Se inicia con una motivación a los estudiantes para que se enfrenten al problema por sí mismos y de forma grupal. Aquí se dan varias subfases:

- Apropiación del problema,

- formulación de estrategias-hipótesis-procedimientos y

- resolución del problema o investigación estudiantil.

El docente tiene un rol activo de acompañamiento, sin dar posibles caminos o estrategias, plantea preguntas generadoras que orienten al estudiantado. Preguntas respecto a la validez y generalización de las hipótesis que pudieran surgir de los estudiantes. Las preguntas se han establecido de forma anticipada en el plan de lección.

\section{Discusión interactiva y comunicativa (15 minutos)}

Posterior al trabajo independiente, se abre un espacio de discusión en donde se exponen diferentes estrategias utilizadas e identificadas por el docente en la fase anterior. Aquí se deben potenciar procesos matemáticos de argumentación y comunicación.

\section{Clausura o cierre (20 minutos)}

A partir de los insumos obtenidos en la fase de discusión, se precisan los conocimientos matemáticos que se querían desarrollar en el proceso. En este caso, se precisó el concepto de bisectriz de un ángulo y la propiedad que se exploró. 


\section{Selección de técnicas de recolección de información}

En este caso se consideraron dos estrategias: videograbación de las lecciones y recolección de información por medio de las hojas de trabajo.

Las videograbaciones se realizaron previo consentimiento por escrito de los padres o encargados de los menores. Solo dos estudiantes no participaron de la grabación por no contar con el permiso de sus padres.

Respecto a la hoja de trabajo, aunque se suministró a cada estudiante el instrumento, se solicitó solo la entrega de una sola hoja por grupo, en la cual se reflejara la solución y respuesta grupal de cada una de las tareas matemáticas.

\section{Implementación}

La puesta en práctica de los planes de lección se realizó con un grupo de sétimo año (primer nivel de educación secundaria) del Colegio Técnico Profesional de Pacayas ubicado en el cantón de Alvarado en la zona noreste de la provincia de Cartago, Costa Rica. Participaron de la lección 29 estudiantes con edades entre los 12 y 14 años, 12 mujeres y 17 hombres. Solo tres estudiantes del grupo estaban repitiendo por primera vez sétimo año, por lo que se puede decir que casi $90 \%$ de los estudiantes que fueron parte del estudio estuvieron en educación primaria el año anterior.

La estrategia metodológica implementada es la principal que propone en el currículo oficial de Matemática, por lo que la dinámica no debería ser extraña para los estudiantes. Aunque no se indagó respecto a si este tipo de organización de lección es habitual en las clases de Matemática, los estudiantes se desenvolvieron correctamente y de forma natural en cada momento o fase de este estilo de lección.

Se formaron tres grupos de tres estudiantes y cinco de cuatro para realizar el trabajo; siguiendo una de las indicaciones metodológicas del Programa de Estudios del MEP (2012, p. 63): "A través del trabajo en grupo se puede fomentar la participación activa. Este consume más tiempo de las lecciones, pero bien realizado permite que haya una adecuada apropiación de los aprendizajes".

\section{Análisis de la información}

Se llevó a cabo una reflexión integral y holística a partir de la revisión de los videos registrados de las lecciones y de las hojas de trabajo de los grupos de estudiantes. Para analizar las respuestas de los estudiantes en cuanto al grado de activación de los procesos, y poder contrarrestarlas respecto a nivel de complejidad de las tareas matemáticas, se empleó el modelo propuesto por Ruiz (2018); debido a que este contempla los elementos teóricos y prácticos de la fundamentación teórica de los programas de estudio del MEP (2012).

En el caso de la primera tarea matemática, el nivel de complejidad se clasifica como Conexión, debido a que en dos procesos se identifican cuatro indicadores en grado 2 , como se muestra en la tabla 2. 
TABLA 2

Estructura de intervención de procesos matemáticos primera tarea matemática del problema

Procesos - capacidades

Razonary argumentar
Indicadores

RA2.1 Identificar información matemática que no está expuesta de manera explícita en una situación matemática o de contexto.

RA2.2 Responder a preguntas donde la respuesta no es directa y amerita mayor argumentación (por ejemplo: ¿cómo hallamos?, ¿qué tratamiento matemático damos?, ¿qué puede o no puede pasar y por qué?, ¿qué sabemos?, ¿qué queremos obtener?).

RA2.5 Elaborar argumentos basados en sus propias acciones al resolver problemas similares a los ya estudiados.

Resolver y plantear problemas

PRP1.2 Resolver problemas que involucran la utilización de algoritmos, fórmulas, procedimientos, propiedades, o convenciones elementales.

Conectar

C1.2 Relacionar conceptos o procedimientos matemáticos distintos dentro de una misma área matemática en la resolución de problemas.

Comunicar

COM2.3 Describir mediante un lenguaje matemáticamente no preciso las acciones, resultados y razonamientos efectuados en la solución de un problema.

Representar Fuente: Elaboración propia a partir de los indicadores de Ruiz (2018, pp. 120-127).

Para la segunda tarea, el nivel de complejidad es de Reflexión, debido a que en dos procesos se identifican tres indicadores en grado 3, como se muestra en la tabla 3 de la estructura de intervención de procesos.

\section{TABLA 3}

Estructura de intervención de procesos matemáticos segunda tarea matemática del problema

\section{Procesos - capacidades}

Razonar y argumentar

Resolver y plantear problemas

Conectar

Comunicar

Representar

\section{Indicadores}

RA3.1 Realizar argumentos matemáticos para resolver problemas o describir situaciones (matemáticas o de contexto real) no estudiados y complejos.

RA3.3 Generalizar los métodos matemáticos utilizados o los resultados obtenidos en la resolución de problemas.

PRP3.2 Generalizar los resultados obtenidos en la resolución de problemas.

C1.2 Relacionar conceptos o procedimientos matemáticos distintos dentro de una misma área matemática en la resolución de problemas.

COM2.3 Describir mediante un lenguaje matemáticamente no preciso las acciones, resultados y razonamientos que ha efectuado en la solución de un problema.

R2.1 Interpretar y razonar sobre la información codificada en una representación matemática dada.

Fuente: Elaboración propia a partir de los indicadores de Ruiz (2018, pp. 120-127).

Se debe subrayar que un mismo problema puede servir a propósitos distintos según las condiciones estudiantiles, por ejemplo, si ya se conociera la propiedad que se quería descubrir en el problema, la segunda tarea matemática se trataría más bien de un ejercicio de reforzamiento o de la aplicación sencilla de un conocimiento, y su estructura de intervención de procesos matemáticos variaría, así como su nivel de complejidad.

\section{DISCUSIÓN DE LOS RESULTADOS}

Lo primero a resaltar es que la totalidad de los grupos mostraron una estrategia inductiva para ambas preguntas que se formularon. Buscaron medidas para los ángulos a y $\beta$ de tal forma que cumplieran con las condiciones geométricas planteadas en la representación geométrica (ver figura 2). Según Isoda 
y Katagiri (2016) en el pensamiento (razonamiento) inductivo los estudiantes primero a partir de cierta cantidad de datos (en este caso medidas de ángulos) intentan descubrir reglas o propiedades comunes para inferir que el conjunto que incluye esos datos satisface las reglas y propiedades descubiertas; por último, es necesario confirmar la validez de la generalidad inferida con nuevos datos.

Figura 2. Imagen tomada de una hoja de trabajo del grupo 2. Ángulo FEG

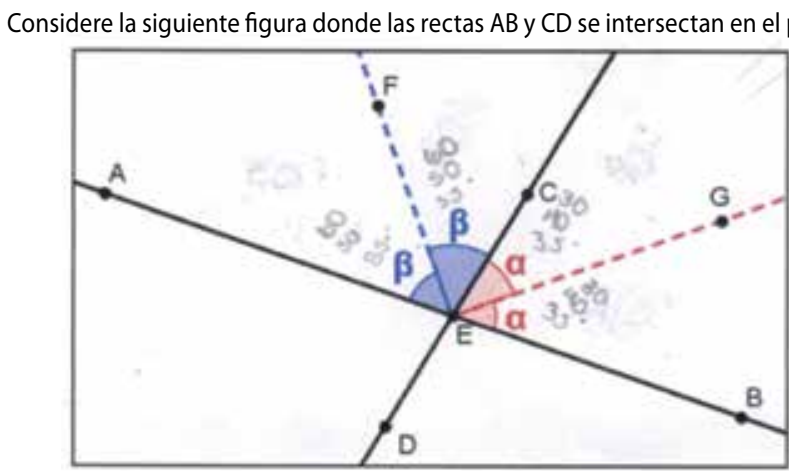

Nota: Se mantiene imagen original según solicitud del autor. Fuente: Elaboración propia (2019).

Esta estrategia le permitió a la mayoría de los grupos conjeturar cuál podría ser la medida del ángulo FEG, ya que estaba compuesto por los ángulos a y $\beta$. En general, los estudiantes pudieron inferir que el ángulo FEG era recto.

La tabla 4 resume y sistematiza la activación de procesos matemáticos evidenciados en esta tarea matemática.

TABLA 4

Activación de procesos matemáticos primera tarea matemática del problema

\begin{tabular}{ccccccccc} 
Indicadores & Grupo 1 & Grupo 2 & Grupo 3 & Grupo 4 & Grupo 5 & Grupo 6 & Grupo 7 & Grupo 8 \\
RA2.1 & ES & ES & ES & ES & ES & ES & ES \\
RA2.2 & EP & ES & EP & NE & EP & EP & EP & NE \\
RA2.5 & EP & ES & NE & NE & EP & EP & NE \\
PRP1.2 & ES & ES & ES & NE & NE & NE & ES & ES \\
C1.2 & ES & ES & ES & ES & EP & EP & ES & EP \\
COM2.3 & EP & ES & NE & EP & EP & EP & EP & NE \\
R2.1 & ES & ES & ES & EP & EP & EP & ES & EP \\
\hline
\end{tabular}

Nota: La codificación es la siguiente ES: Evidencia suficiente, EP: Evidencia parcial, NE: No hay evidencia.

Fuente: Elaboración propia.

En síntesis, existe evidencia suficiente de que los grupos de estudiantes identificaron la información matemática que no se dio de manera explícita en la situación geométrica planteada. Sin embargo, solo el grupo 2 mostró evidencia suficiente respecto a los indicadores RA2.2 y RA2.3 que se relacionan con la elaboración de argumentos que respaldan su respuesta.

En cuanto al indicador PRP1.2 solo en los grupos 4, 5 y 6 no hay evidencia de su activación, además se identificó un error común en estos grupos que fue dar una respuesta a partir de supuestos equivocados. Respecto a los demás procesos matemáticos existe diferente grado de evidencia referente a que utilizan y relacionan los conocimientos previos (par lineal, ángulos congruentes, ángulo recto, etc.) y de que 
describen con sus palabras y algunos signos matemáticos los procedimientos y respuestas. Asimismo, hay evidencia suficiente y parcial que los grupos pudieron interpretar la representación geométrica y sus elementos, y razonar a partir de esta.

En el caso de la segunda tarea matemática, aunque todos los grupos en la primera pregunta pudieron inferir que el ángulo FEG era recto, solo los grupos 1, 2 y 3 respondieron de forma escrita a la segunda pregunta planteada; es decir, en la mayoría de los casos no lograron generalizar los resultados y no realizaron ninguna conjetura de la propiedad que se quería descubrir. Por ejemplo, se muestra esta respuesta, que aunque respondieron correctamente la primera pregunta referente a la medida del ángulo FEG, no pudieron darse cuenta de la generalización del resultado. Aquí lo que se puede interpretar es que no hubo buena comprensión de la pregunta formulada o no se respondió a partir de lo realizado para la pregunta anterior.

Figura 3. Respuestas a la pregunta 2 relacionadas al Problema "Ángulo FEG".

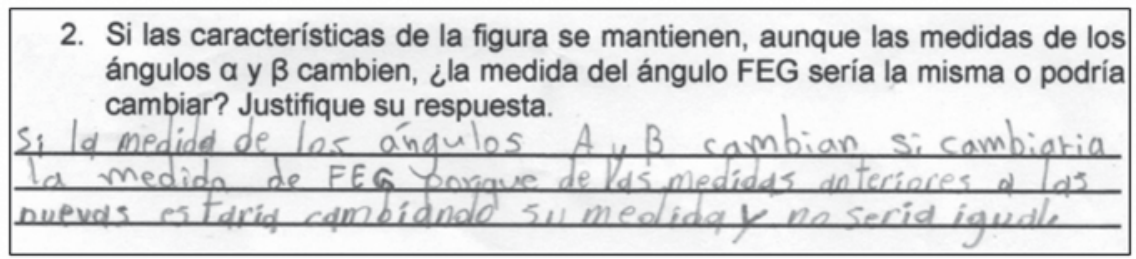

Fuente: Tomado de la hoja de trabajo del grupo 1

Sin embargo, un grupo en particular logró llegar a una generalización, dándose cuenta que el ángulo FEG es la mitad del ángulo llano AEB, y que si la figura conservaba sus condiciones geométricas, el ángulo FEG permanecía invariante.

Figura 4. Respuestas a las preguntas 1 y 2 relacionadas al Problema "Ángulo FEG".

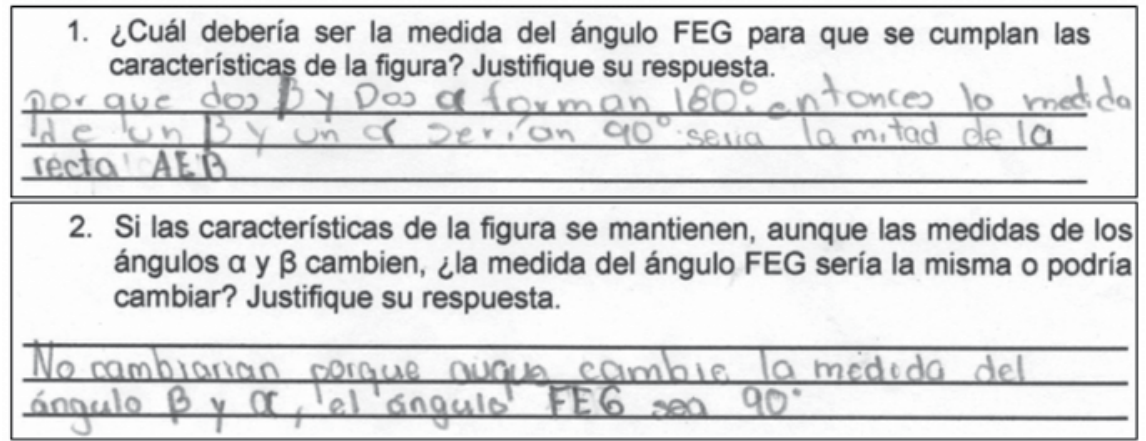

Fuente: Tomado de la hoja de trabajo del grupo 2

Respecto al grado de activación de procesos se hace el siguiente balance: se logró evidenciar en un par de grupos que realizaron argumentos matemáticos para resolver el problema o al menos describir la situación matemática. Sin embargo, solo el grupo 2 mediante razonamiento inductivo pudo lograr una generalización empírica de la propiedad que se quería estudiar.

En general, se notó gran dificultad para poder realizar argumentos verbales (a partir de la videograbación) y escritos utilizando lenguaje matemático (no preciso) para explicar las acciones, resultados y razonamientos realizados en la solución de la tarea matemática. Sin embargo, a pesar de esto se generaron insumos suficientes para la fase de discusión y de clausura. 
Aunque el tratamiento empírico por parte de los estudiantes fue valioso para los diferentes momentos de la lección, esta aproximación mediante la construcción inductiva se acompañó por la formalización y la estructuración de ese conocimiento en la fase de clausura.

En cuanto a la organización de la lección, mediante la videograbación, se pudo evidenciar claramente los cuatro momentos establecidos en MEP (2012), y queda en evidencia el papel del docente en este tipo de metodología de enseñanza: el educador se muestra activo como un facilitador, en términos de guía, orientación y formulación de preguntas apropiadas que le permitieran a los estudiantes enfrentarse al problema de forma independiente sin ofrecer la respuesta o la ruta de solución al problema. Además, el docente le insistió a los estudiantes la necesidad de verificar las conjeturas con nuevos datos, debido a que cuando los resultados se obtienen por medios inductivos estos podrían ser engañosos (Isoda y Katagiri, 2016).

\section{CONCLUSIONES Y RECOMENDACIONES}

En los últimos tiempos, se ha separado la investigación educativa de la práctica docente, creando en muchos casos la poca practicidad de los resultados de investigación al ambiente educativo. Perines (2018) advierte que "la investigación y la práctica educativa avanzan por caminos radicalmente distintos, antagónicos y, por qué no decirlo, casi irreconciliables" (p. 10). Por lo anterior se establece como primera conclusión la importancia de este tipo de investigaciones en un contexto de implementación curricular en donde se introducen novedades teóricas y metodológicas.

Es necesario que la práctica educativa se convierta en un proceso autoreflexivo donde la teoría y la práctica se articulen de forma dinámica; por lo que esta experiencia de aula puede servir de modelo para docentes en servicio y estudiantes en formación, ya que podrían clarificar algunos componentes teóricos del currículo de una forma pragmática.

Respecto al problema analizado, a partir de la estructura de intervención de procesos matemáticos se coloca a la segunda tarea matemática en un nivel de complejidad de Reflexión; y esto coincide con los resultados obtenidos en la aplicación. A su vez, lo anterior está en concordancia con lo establecido en MEP (2012, p. 29): “Un problema debe poseer suficiente complejidad para provocar una acción cognitiva no simple. Si se trata esencialmente de acciones rutinarias, no se conceptuarán como problemas". Asimismo, partiendo de la premisa que una tarea matemática estará determinada por el propósito educativo (Ruiz, 2018), la situación matemática "ángulo FEG" se puede considerar un problema apto para el desarrollo de conocimientos nuevos, ya que busca generar la interrogación y la acción estudiantil utilizando conceptos o métodos matemáticos sin que hayan sido detalladamente explicadas ni se hayan mostrado soluciones similares con anterioridad. Isoda y Olfos (2009, p. 107) señalan que "la clase ha de entenderse como un proceso y el problema como un vacío o diferencia entre un estado actual y uno esperado".

La valoración de una tarea matemática para el aprendizaje de conocimientos nuevos debe centrarse no tanto en si las respuestas fueron correctas o no, sino en los insumos que se pueden generar a partir de la discusión, y que sirven para la formalización de conceptos nuevos en la fase de clausura o cierre. Se debe tener claro que no es lo mismo diseñar una situación para la construcción de aprendizajes que hacerlo para reforzarlos, pues en el segundo caso el estudiante ya tiene elementos para aplicar o movilizar esos aprendizajes. Por ejemplo, hay que comprender que en una tarea para el desarrollo de habilidades matemáticas nuevas, el estudiante cuenta con el apoyo e intervención docente y tiene propósitos distintos a una tarea que pretende la evaluación sumativa. 
Asimismo, como lo que se desea es desarrollar la competencia matemática, es importante centrar la atención en la activación de procesos matemáticos. Los resultados evidencian la activación de procesos como Representar a la hora de interpretar la situación gráfica planteada en el problema; asimismo, el proceso Comunicar, al exponer los razonamientos de forma escrita y verbal. Especialmente se activa el proceso Razonar y argumentar, ya que si bien no se esperaba una prueba formal de la propiedad estudiada, los "insumos" originados con la solución del problema pueden considerarse una fase previa al desarrollo de la demostración formal.

Un aspecto por destacar fue que los estudiantes optaron por el razonamiento inductivo en lugar del deductivo. Muchas veces se desmerita este tipo de razonamiento debido a que la formación matemática docente ha favorecido el razonamiento deductivo. Polya (1979) destaca el papel del razonamiento inductivo sobre el deductivo en la enseñanza y aprendizaje de las matemáticas al indicar que "la matemática presentada con rigor son una ciencia sistemática, deductiva, pero las matemáticas en gestación son una ciencia experimental, inductiva" (p. 114). Para problemas de nivel de complejidad alto como este, donde se requiere una generalización por parte de los estudiantes, el análisis de patrones geométricos y la descripción de sus regularidades y propiedades promueve capacidades de abstracción, visualización y razonamiento flexible (Sriraman, 2004 y Vogel, 2005).

Por último, a partir del estudio de la lección por medio del video se concluye que el problema cumplió con los propósitos establecidos para la lección. Queda claro que una selección acertada o diseño adecuado del problema es la clave para el éxito de una clase enmarca en la resolución de problemas como herramienta metodológica. Isoda y Olfos (2009, p. 100) subrayan: "La selección y estudio de buenos problemas es una tarea compleja y valiosa en la didáctica de la matemática"; además, estos autores destacan que a partir de una buena escogencia del problema se pueden establecer de manera natural los demás elementos del plan de lección; es decir, el problema debe ser el punto de partida en la elaboración de un plan de lección.

\section{REFERENCIAS}

Blanco, A., Martínez, B. y Jiménez, M. (2018). ¿Puede la investigación iluminar el cambio educativo? Ápice. Revista de Educación Científica, 2(2), 15-28. DOI: https://doi.org/10.17979/arec.2018.2.2.4612

Bransford, J. y Stein, B. (1987). Solución IDEAL de Problemas. Barcelona, España. Ed. Labor.

Cerrón, W. (2019). La investigación cualitativa en educación. Horizonte de la Ciencia, 9(17). DOI:10.26490/ uncp.horizonteciencia.2019.17.510

Díaz, J. y Díaz, R. (abril, 2018). Los Métodos de Resolución de Problemas y el Desarrollo del Pensamiento Matemático. Bolema, 32 (60), 57-74. Recuperado de https://doi.org/10.1590/1980-4415v32n60a03

Gil, J., León, J. y Morales, M. (2017). Los paradigmas de investigación educativa, desde una perspectiva crítica. Revista Conrado, 13(58), 72-74. Recuperado de https://conrado.ucf.edu.cu/index.php/ conrado/article/view/476/510

Guzmán, M. (1991). Para pensar mejor. Barcelona, España. Ed. Labor.

Hernández, L. y López, E. (2015). Modelos de planeamiento didáctico y plan de lección empleando la Integración de habilidades Matemáticas para la Educación Secundaria. Cuadernos de Investigación y formación en educación matemática, 13, 289-299. Recuperado de http://revistas.ucr.ac.cr/index. php/cifem/article/view/19163/19218

Isoda, M. y Olfos, R. (2009). El enfoque de resolución de problemas. Ediciones universitarias de Valparaíso. Valparaíso, Chile. 
Isoda, M. y Katagiri, S. (2016). Pensamiento matemático: Cómo desarrollarlo en la sala de clases. Centro de Investigación Avanzada en Educación, Universidad de Chile. Chile.

Isoda, M., Arcavi, A. y Mena, A. (2012). El estudio de clases japonés en Matemáticas. Ediciones universitarias de Valparaíso. Valparaíso, Chile.

Mason, J. Burton, K. y Stacey, K. (1982). Pensar Matemáticamente. Barcelona, España. Ed. Labor.

Mazzilli, D., Hernández, L. y De La Hoz S. (2016). Procedimiento para Desarrollar la Competencia Matemática Resolución de Problemas. Escenarios, 14 (2), 103-119 DOI: http://dx.doi.org/10.15665/ esc.v14i2.935

Meza, L. Agüero, E. y Suarez, Z. (agosto, 2019). Reforma de la educación matemática en Costa Rica: evaluación de avance de la implementación en la educación secundaria. Matemática, Educación e Internet. 19(2). ISSN 1659 -0643. Recuperado de https://tecdigital.tec.ac.cr/revistamatematica/ ARTICULOS_V19_N2_2019/RevistaDigital_MezaAgueroSuarez_V19_n2_2019/RevistaDigital_ MezaAgueroSuarez_V19_n2_2019.pdf

Ministerio de Educación Pública (MEP) (2012). Programas de Estudio de Matemáticas. I, II Y III Ciclos de la Educación General Básica y Ciclo Diversificado. San José: Autor.

Perines, H. (2018). ¿Por qué la investigación educativa no impacta en la práctica docente? Estudios sobre educación, 34, 9-27. Recuperado de https://doi.org/10.15581/004.34.9-27

Polya, G. (1945). How to solve it. A new aspect of mathematical method. Princeton University Press.

Polya, G. (1979). Cómo plantear y resolver problemas [Obra original publicada en 1945]. México: Trillas.

Programa Estado de la Nación. (2015). Estado de la educación costarricense. San José, Costa Rica: Editorial ED S.A.

Ruiz, A. (2018). Evaluación y pruebas nacionales para un currículo de Matemáticas que enfatiza capacidades superiores. México: Comité Interamericano de Educación Matemática CIAEM. ISBN 978-607-97480-3-6

Schoenfeld, A. (1985). Mathematical Problem Solving. New York: Academic Press.

Sriraman B. (2004). Reflective abstraction, uniframes and the formulation of generalizations. Journal of Mathematical Behavior, 23, 205-222.

Vargas, Z. (2009). La investigación aplicada: una forma de conocerlas realidades con evidencia científica. Revista Educación, 33(1), 155-165.

Villegas, J. L., Castro, E., y Gutiérrez, J. (2009). Representaciones en resolución de problemas: un estudio de caso con problemas de optimización. Electronic Journal of Research in Educational Psychology, 7(17), 279-308. Recuperado de http://www.investigacionpsicopedagogica.org/revista/articulos/17/espannol/Art_17_297.pdf

Vogel R. (2005). Patterns: A fundamental idea of mathematical thinking and learning. ZDM International Journal on Mathematics Education, 37(5), 445-449.

Yerushalmy, M. (2000). Problem solving strategies and mathematical resources: a longitudinal view on problem solving in a function based approach to algebra. Educational Studies in Mathematics, 43(2), 125-147, doi: 10.1023/A:1017566031373

Yimer, A. y Ellerton, N. (2010). A five-phase model for mathematical problem solving: Identifying synergies in pre-service teachers' metacognitive and cognitive actions. ZDM The International Journal on Mathematics Education, 2(42), 245-261, doi: 10.1007/s11858-009-0223-3

Zumbado, M., Oviedo, D. y Vargas, G. (2015). Integración de habilidades matemáticas en el plan de lección y el planeamiento del periodo, modelo para la Educación Primaria. Cuadernos de Investigación y formación en educación matemática, 13, 275-287. Recuperado de http://revistas.ucr.ac.cr/index. $\mathrm{php/cifem/article/view/19162/19220}$ 
ANEXO A:

Problema "Ángulo FEG"

Ángulo FEG

Considere la siguiente figura donde las rectas $A B$ y $C D$ se intersectan en el punto $E$ :

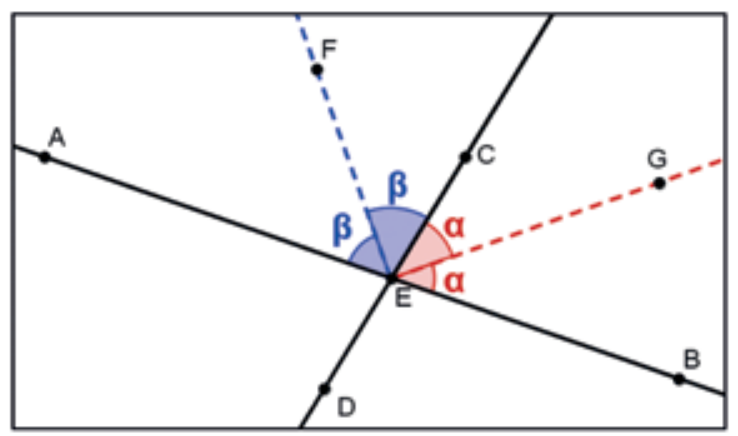

De acuerdo a la figura anterior, responda lo siguiente:

1. ¿Cuál debería ser la medida del ángulo FEG para que se cumplan las características de la figura? Justifique su respuesta.

2. Si las características de la figura se mantienen, aunque las medidas de los ángulos a y $\beta$ cambien, ¿la medida del ángulo FEG sería la misma o podría cambiar? Justifique su respuesta. 
ANEXO B:

\section{Modelo de una clase basada en el método de resolución de problemas}

\section{Comprensión del problema Aproximadamente \\ 10 minutos}

- Lectura atenta del texto del problema y comprensión de la situación planteada.

- Aclaración de la situación problema presentando atención a las indicaciones del profesor y mediante la discusión entre los niños.

- Comparación (similitudes y diferencias) entre lo estudiado anteriormente y el presente problema.

- Proposición de primeras sugerencias de resolución y respuestas.

Fuente: Isoda, M., Arcavi, A. y Mena, A. (2012, p. 117).

Para citar este documento:

Hernández-Solís, L. (2020). Diseño y desarrollo de lecciones mediante el enfoque del currículo costarricense de Matemática. Revista Innovaciones Educativas. 22(32), 7-20. DOI: 10.22458/ ie.v22i32.2767 